\title{
The Neurocritical Care Society of India (NCSI) and the Indian Society of Neuroanaesthesiology and Critical Care (ISNACC) Joint Position Statement and Advisory on the Practice of Neurocritical Care during the COVID-19 Pandemic
}

\author{
Ponniah Vanamoorthy ${ }^{1}$ Gyaninder P. Singh ${ }^{2, \odot}$ Prasanna U. Bidkar ${ }^{3}$ Ranadhir Mitra ${ }^{4}$ \\ Kamath Sriganesh ${ }^{5}$ Siddharth Chavali6, ${ }^{6}$ Radhakrishnan Muthuchellapan ${ }^{5}$ Venkatesh H. Keshavan \\ Saurabh Anand ${ }^{8}$ Keshav Goyal ${ }^{2}$ Rahul Yadav ${ }^{9}$ Girija P. Rath ${ }^{2, \odot ~ S h a s h i ~ S r i v a s t a v a ~}{ }^{10}$
}

\footnotetext{
${ }^{1}$ Department of Neuroanaesthesiology and Neurocritical Care, Institute of Neurosciences and Spinal Disorders, MGM Healthcare, Chennai, Tamil Nadu, India

${ }^{2}$ Department of Neuroanaesthesiology and Critical Care, All India Institute of Medical Sciences, New Delhi, India

${ }^{3}$ Division of Neuroanaesthesiology, Department of Anesthesiology and Critical care, Jawaharlal Institute of Post Graduate Medical Education and Research, Puducherry, India

${ }^{4}$ Department of Neuroanesthesia and Neurocritical Care, Care Hospital, Bhubaneswar, Odisha, India

${ }^{5}$ Department of Neuroanaesthesiology and Neurocritical Care, National Institute of Mental Health and Neuro-Sciences, Bengaluru, Karnataka, India

6Department of Neurosciences, Aditya Birla Memorial Hospital, Pune, Maharashtra, India
}

\author{
Address for correspondence Gyaninder P. Singh, Department of \\ Neuroanaesthesiology and Critical Care, Room No. 711 (7th Floor), \\ Neurosciences Centre, All India Institute of Medical Sciences, \\ New Delhi, India (e-mail: drsingh_gp@yahoo.co.in).

\footnotetext{
${ }^{7}$ Department of Neuroanaesthesiology and Neurocritical Care, Apollo Hospitals, Bengaluru, Karnataka, India

${ }^{8}$ Department of Neuroanaesthesia and Neurocritical Care, Artemis Hospital, Gurugram, Haryana, India

${ }^{9}$ Department of Anaesthesiology and Critical Care, INHS Asvini, Mumbai, Maharashtra, India

${ }^{10}$ Department of Anesthesiology and Critical Care, Sanjay Gandhi Post Graduate Institute of Medical Sciences, Lucknow, Uttar Pradesh, India
}

\author{
Abstract \\ Keywords \\ - COVID-19 pandemic \\ - SARS-CoV-2 \\ - neurological \\ manifestations \\ - critically ill \\ neurological patients \\ - neurocritical care \\ management
}

The COVID-19 pandemic caused by severe acute respiratory syndrome coronavirus (SARS-CoV-2) has rapidly spread across the world including India. Management of patients complicated with neurological illness requiring neurocritical care is challenging during this time. Patients with neurological disease may develop COVID-19 infection or there could be independent neurological manifestations of COVID-19. Critically ill neurological patients are more vulnerable to contracting SARS-CoV-2 infection. Also, neurological patients with comorbidities and multisystem involvement are at increased risk of adverse outcomes. Though SARS-CoV-2 predominantly affects the pulmonary system, it can complicate the assessment and management of neurological patients. With increasing COVID-19 numbers, the hospitalizations of both non-COVID and COVID-19 neurological patients will bring significant strain on the
DOI https://doi.org/ $10.1055 / \mathrm{s}-0040-1714648$ ISSN 2348-0548.
(C) 2020. Indian Society of Neuroanaesthesiology and Critical Care. This is an open access article published by Thieme under the terms of the Creative Commons Attribution-NonDerivative-NonCommercial-License, permitting copying and reproduction so long as the original work is given appropriate credit. Contents may not be used for commercial purposes, or adapted, remixed, transformed or built upon. (https://creativecommons.org/licenses/by-nc-nd/4.0/). Thieme Medical and Scientific Publishers Pvt. Ltd., A-12, 2nd Floor, Sector 2, Noida-201301 UP, India 
hospital and neurocritical care facilities. Streamlining work pattern, understanding the pathophysiology of COVID-19 and its impact on neurological function, establishing general and specific neurocritical care management strategies, ensuring protection and well-being of health care providers, and implementing effective infection control policies are key elements of efficient neurocritical care management during this pandemic. This joint position statement and advisory on the practice of neurocritical care during the COVID-19 pandemic by the Neurocritical Care Society of India and the Indian Society of Neuroanaesthesiology and Critical Care has been developed to guide clinicians providing care to the critically ill neurological patients in the neurocritical care unit during the current pandemic. As the situation from this novel disease is rapidly evolving, readers must constantly update themselves with newly emerging evidence to provide the best possible care to the critically ill neurological patients.

\section{Introduction}

The pandemic of coronavirus disease 2019 (COVID-19) has rapidly spread throughout the world leading to a global health crisis. The fast-rising numbers of COVID-19 patients has stressed the health care system. The management of non-COVID patients is also significantly affected as the limited health care resources are diverted toward managing the patients with COVID-19. Medical emergencies including neuro-emergencies continue unabated during this period and require immediate care to save lives and avoid irreversible consequences. Neurocritical care involves time-sensitive interventions where rapid workup and prompt management improve patient outcomes. Management of these patients should not be delayed for lack of test for COVID-19.

A patient with neurological disease may acquire COVID-19 infection, or neurological illness may be a manifestation of COVID-19. Management of such patients poses unique challenges. These patients need isolation and critical care in dedicated COVID intensive care units (ICUs). Currently, there is limited data to guide neurocritical care unit (NCCU) management of patients with suspected/confirmed COVID-19 infection. The purpose of this position statement and advisory is to guide clinicians on the practice of neurocritical care during the ongoing COVID-19 pandemic.

\section{Methodology}

The Governing Councils of the Neurocritical Care Society of India and the Indian Society of Neuroanaesthesiology and Critical Care formed an expert committee of neurointensivists from across India to formulate this advisory. A detailed search of PubMed, Embase, Medline, OVID, and Google Scholar databases were done for the available information from original investigations, case series, and special articles. The search included these specific words: COVID-19 and neurocritical care, neurological emergencies, stroke, spinal cord injury, and mechanical ventilation. Information was also obtained from the guidelines and practice advisories of allied specialties of neurology, neurosurgery, and intensive care.
Additional information was retrieved from the references of these articles.

Directives from the Ministry of Health and Family Welfare (MoHFW), Government of India and the Indian Council of Medical Research were obtained from respective Web sites. Since definitive evidences were not available, the committee members contacted experts in the field of neurocritical care across India to obtain their views. The expert committee held multiple web-based discussions to formulate this consensus statement.

\section{General Principles of Management during COVID-19}

\section{Triage}

The neurocritical care team should work closely with the emergency department and the COVID-19 team to screen, test, and isolate suspected COVID-19 patients with critical neurological illness contemplating admission. Symptoms described as influenza-like illness (ILI) such as fever (temperature $>38^{\circ} \mathrm{C}$ ) and cough are typical manifestations of COVID-19 and it is recommended to isolate these patients in the screening area itself. Apart from these symptoms, patients may present with atypical manifestations like myalgia, sore throat, alteration in taste or smell, diarrhea, and cardiovascular manifestations. Many of the COVID-19 patients may have low oxygen saturation without dyspnea (silent hypoxia). The diabetic and older patients with COVID-19 are more likely to suffer silent hypoxia. ${ }^{1}$ Confirmed COVID-19 patients, irrespective of the symptoms, should be managed in a dedicated COVID ICU. If a hospital does not have such a facility, they should transfer the patient to a designated COVID-19 facility after resuscitation and stabilization. The COVID-19 suspects (patients with ILI, fever, exposure to high-risk contacts, and from containment zones) should be isolated and cared for in an isolation ICU till the reverse transcriptase polymerase chain reaction (RT-PCR) results are available (-Fig. 1). As approximately $20 \%$ of RT-PCR results are false negative such patients with severe acute respiratory illness (SARI), with no other explainable cause, should continue to receive care in 


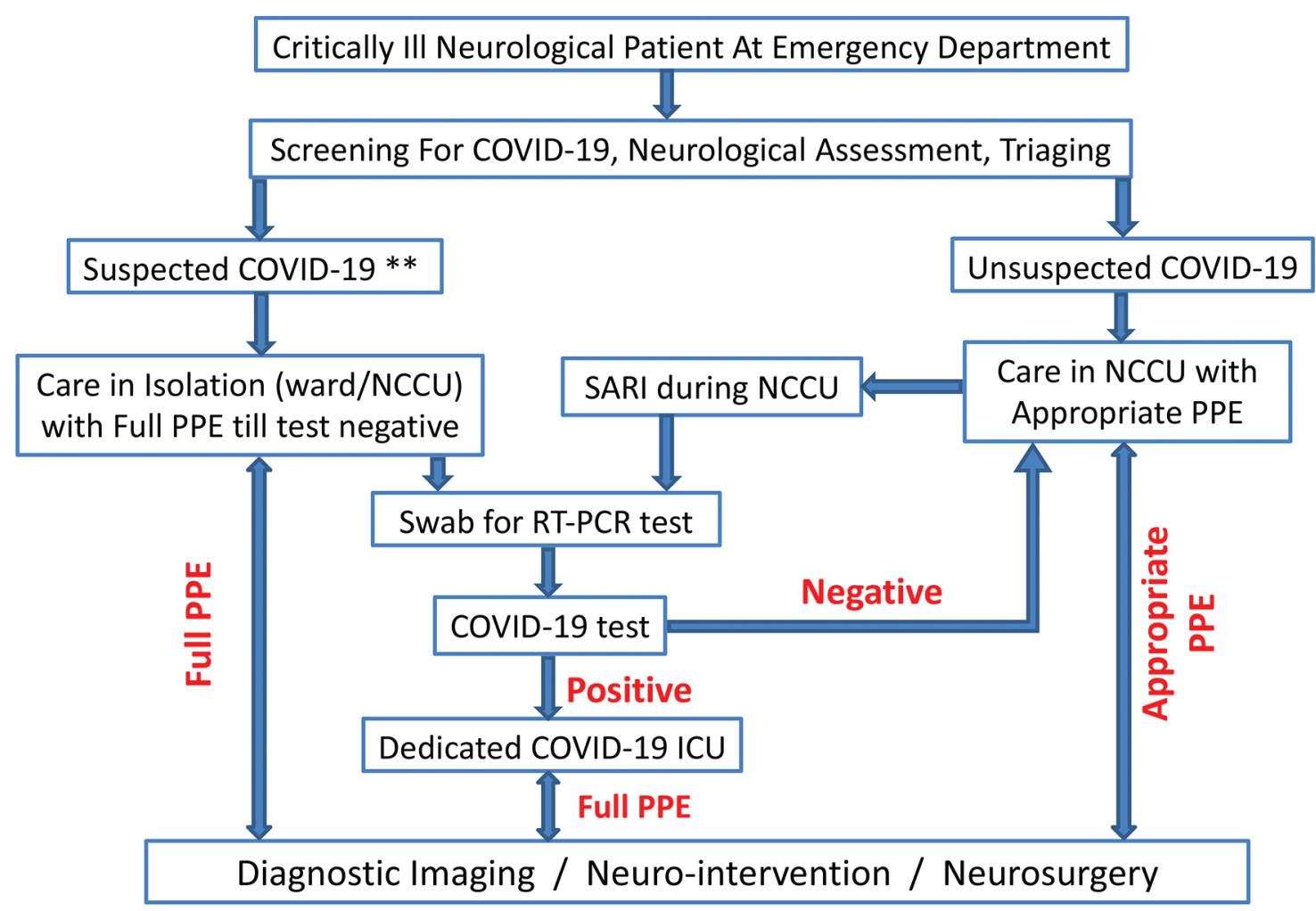

\section{** Includes symptomatic cases and asymptomatic patients from containment areas $\longleftrightarrow$ Transport; NCCU= Neurocritical Care Unit; SARI= Severe Acute Respiratory Illness; $\mathrm{PPE}=$ Personal Protective Equipment; RT-PCR= Reverse Transcriptase Polymerase Chain Reaction}

Fig. 1 Triage and transport of critically ill neurological patients during management in the hospital.

isolation and should undergo repeat test. ${ }^{2}$ Any patient admitted with critical neurological illness who develops SARI later, should also be isolated and investigated for COVID-19.

\section{Preparedness of Neurocritical Care Services and Surge Response}

The numbers of COVID-19 cases in India are currently increasing. At the same time, neurologic emergencies such as acute ischemic stroke (AIS), intracranial hemorrhage $(\mathrm{ICH})$, traumatic brain injury (TBI), spinal cord injury (SCI), and aneurysmal subarachnoid hemorrhage (aSAH) continue to present to NCCU. Additionally, COVID-19 patients who develop acute neurologic illness such as viral meningitis, encephalitis, or Guillain-Barre syndrome (GBS) may require NCCU admission. Moreover, patients with autoimmune disorders such as multiple sclerosis, myasthenia gravis (MG), and sarcoidosis, who receive immunosuppressive therapies, are more prone to COVID-19 infection. Thus, there will be an increased requirement for neurocritical care support which necessitates optimization of resources during the pandemic. ${ }^{3}$ This requires innovative measures with regards to space, staff, supplies, and equipment. Emphasis should be on reorganizing health care teams, enhancing hospital and ICU capacity, and acquisition of personal protective equipment (PPE) and ventilators. Focusing on safety and resilience of health care professionals (HCPs) during pandemic-related surge is essential to enhance disaster preparedness. ${ }^{4}$

Some of the strategies to scale-up preparedness in this situation include:

- Using clearly defined clinical management algorithms for neurological emergencies. This limits exposure of team member, while ensuring optimal patient care.

- Creating separate care areas within the NCCU (isolation ICU within ICU) for treating suspected and confirmed COVID-19 patients till test results are available.

- HCPs should work in a safe environment, with adequate PPEs and appropriate training in its use.

- Video-laryngoscope should be available for airway management.

- Provision of tiered staffing plan with availability of reserve team and alternate staffing resources.

- Adopting electronic-ICU monitoring (remote patient monitoring by intensivists at an offsite facility) to support on-site staff. Utilizing telemedicine and videoconferencing for taking rounds and consultations, and for training and education of trainees and staff.

- Providing ready access to hospital isolation guidelines and testing protocols.

- Providing access to psychological support to worried, anxious, and stressed staff. 
The World Health Organization (WHO) has developed the Adaptt Surge Planning Support Tool to support surge planning. ${ }^{5}$ It is accompanied by technical guidance which outlines actions and policies to increase available hospital capacity for an influx of COVID-19 patients, while continuing to maintain essential services.

\section{Environmental Cleaning and Disinfection}

During COVID-19, cleaning and disinfection becomes more important to control the pandemic.

(1) Cleaning: The surfaces should be thoroughly scrubbed with water and soap or detergent.

(2) Disinfection: Various agents have viricidal activity when used in right concentration, for right contact time, and in right amount. A list of these agents is available from Environmental Protection Agency USA, ${ }^{6}$ MoHFW India, ${ }^{7-9}$ and National Centre for Disease Control. ${ }^{10}$

\section{Personal Protective Equipment in Neurocritical Care}

The MoHFW has provided guidelines for the appropriate and rational use of PPE in different care areas (- Table 1). ${ }^{11,12}$ All HCPs should use appropriate PPE depending on the work area and risk profile and also appropriately dispose used PPE according to guidelines..$^{13}$ Standard precautions should always be followed as PPEs are not alternative to basic preventive public health measures such as hand hygiene and respiratory etiquettes.

\section{Donning and Doffing}

Proper donning (wearing) and doffing (removing) of PPE is crucial to prevent infection transmission among HCPs. The procedure of donning and doffing should be followed as per instructions from Centers for Disease Control and Prevention ${ }^{14}$ and WHO. ${ }^{15}$ HCPs are at increased risk of infection during doffing; hence, it should always be supervised. Any breach in the PPE or procedure should be reported immediately to the concerned authority.

\section{Transport of Patient to and from Neurocritical Care Unit}

All persons involved in transport of suspected/confirmed COVID-19 patients should don appropriate PPE before shifting the patient. All procedures and investigations for these patients should preferably be performed at bedside and transport within the hospital should be avoided

Table 1 The recommendations for PPE based on the work area and risk profile for HCPs

\begin{tabular}{|c|c|c|c|c|c|}
\hline No. & Location & Activity & Risk & Recommended PPE & Remarks \\
\hline 1 & Triage area & Triaging patients & Moderate & N95 mask, gloves & $\begin{array}{l}\text { Patients to wear triple } \\
\text { layered mask }\end{array}$ \\
\hline \multirow[t]{2}{*}{2} & \multirow{2}{*}{$\begin{array}{l}\text { Transporting } \\
\text { patients to/from } \\
\text { NCCU to other } \\
\text { areas of hospital } \\
\text { like ED, radiology, } \\
\text { OR }\end{array}$} & $\begin{array}{l}\text { Transporting patients } \\
\text { not on any assisted } \\
\text { ventilation }\end{array}$ & Low & $\begin{array}{l}\text { Triple layer medical } \\
\text { mask, Latex } \\
\text { examination gloves }\end{array}$ & $\begin{array}{l}\text { No AGPs } \\
\text { (patients to wear triple } \\
\text { layered mask) }\end{array}$ \\
\hline & & $\begin{array}{l}\text { SARI patient with } \\
\text { or without assisted } \\
\text { ventilation }\end{array}$ & High & $\begin{array}{l}\text { Full complement of } \\
\mathrm{PPE}^{\mathrm{a}}\end{array}$ & Potential AGPs \\
\hline \multirow[t]{3}{*}{3} & \multirow[t]{3}{*}{$\begin{array}{l}\text { NCCU } \\
\text { (non-COVID area) }\end{array}$} & $\begin{array}{l}\text { Critical care } \\
\text { management }\end{array}$ & Moderate & $\begin{array}{l}\text { N95 mask, goggles, } \\
\text { nitrile examination } \\
\text { gloves + face shield }\end{array}$ & $\begin{array}{l}\text { Potential AGPs } \\
\text { Face shield, when a splash } \\
\text { of body fluid is expected }\end{array}$ \\
\hline & & $\begin{array}{l}\text { Critical care } \\
\text { management } \\
\text { (patient develop fever or } \\
\text { SARI during NCCU stay) }\end{array}$ & High risk & $\begin{array}{l}\text { Full complement of } \\
\text { PPE }^{a}\end{array}$ & $\begin{array}{l}\text { Isolation in NCCU (ICU } \\
\text { within ICU concept) till the } \\
\text { RT-PCR results }\end{array}$ \\
\hline & & $\begin{array}{l}\text { Dead body packing/ } \\
\text { transport to mortuary }\end{array}$ & Low & $\begin{array}{l}\text { Triple layered medical } \\
\text { mask, latex gloves }\end{array}$ & No AGPs \\
\hline \multirow[t]{4}{*}{4} & \multirow[t]{4}{*}{ COVID ICU } & $\begin{array}{l}\text { Critical care } \\
\text { management }\end{array}$ & High & $\begin{array}{l}\text { Full complement of } \\
\text { PPE }^{a}\end{array}$ & Potential AGPs \\
\hline & & $\begin{array}{l}\text { Cardiopulmonary } \\
\text { resuscitation }\end{array}$ & High & $\begin{array}{l}\text { Full complement of } \\
\mathrm{PPE}^{\mathrm{a}}\end{array}$ & Potential AGPs \\
\hline & & Dead body packing & High & $\begin{array}{l}\text { Full complement of } \\
\text { PPE }^{\mathrm{a}}\end{array}$ & No AGPs \\
\hline & & $\begin{array}{l}\text { Dead body transport to } \\
\text { mortuary }\end{array}$ & Low & $\begin{array}{l}\text { Triple layered medical } \\
\text { mask, latex gloves }\end{array}$ & No AGPs \\
\hline
\end{tabular}

Abbreviations: AGPs, aerosol-generating procedures; ED, emergency department; HCP, health care professionals; ICU, intensive care unit; NCCU, neurocritical care unit; OR, operating room; PPE, personal protective equipment; RT-PCR, reverse transcriptase polymerase chain reaction; SARI, severe acute respiratory illness.

${ }^{\mathrm{a}}$ Full complement of PPE = N95 mask, coverall, nitrile/latex examination gloves, shoe cover, eye goggles (with or without face shields). 
unless absolutely necessary. During transport, a dedicated shortest route corridor with separate entry and exit for COVID-19 patients is preferable to reduce transmission of infection. Else, the entire pathway should be disinfected after transport. Intubated patients on mechanical ventilation should have a viral filter attached between the endotracheal tube (ETT) and the circuit while all nonintubated patients should wear a triple-layer surgical mask while being transported.

Before the arrival of suspected/confirmed COVID-19 patient to the NCCU, the following preparations and precautions are suggested:

(1) Prepare negative pressure room (NPR), if possible.

(2) The attending team should don appropriate PPE.

(3) Nonintubated patient must wear a surgical mask and receive supplemental oxygen if $\mathrm{SpO}_{2}$ is $<92 \%$ via a facemask placed over the surgical mask.

(4) For intubated patients, one viral filter is positioned between the ETT and the ventilator circuit, and another at the expiratory port of the ventilator.

(5) Intubated patients should have closed suction system to minimize circuit disconnection.

(6) Necessary precautions should be taken during any aerosol-generating procedures (AGPs).

(7) If surgery is planned in suspected/confirmed COVID-19 patient, the NCCU team should inform the operation theater personnel in advance.

(8) For all COVID-19 suspects, nasopharyngeal swab for RT-PCR test should be sent at the earliest. The patient should be considered as positive till the results are obtained.

\section{Management in Neurocritical Care Unit}

\begin{abstract}
Airway Management
Airway management is a crucial part of care in critically ill neurological patients. Handling of airway is an AGP, which predisposes HCPs to the risk of infection. One in $10 \mathrm{HCPs}$ contracted COVID-19 during intubation of suspected/confirmed COVID-19 patients despite wearing PPE conforming to WHO standards. ${ }^{16}$ Various AGPs performed in the NCCU are intubation and extubation, bronchoscopy, open suctioning, nebulization, manual ventilation, disconnection of patient from the ventilator, noninvasive positive pressure ventilation (NIPPV), tracheostomy, prone positioning, and cardiopulmonary resuscitation (CPR). In suspected/confirmed COVID-19 patient, we recommend the following:
\end{abstract}

1. AGPs in the NCCU should be performed in NPR (if available) to prevent spread of contagious airborne pathogens outside the room. WHO recommends the use of NPR with at least 12 air changes per hour. ${ }^{17}$

2. Minimum number of personnel should remain inside the room during AGPs. ${ }^{18}$

3. Intubation should be performed by an experienced person to maximize the chances of success, and minimize the number of attempts. Rapid-sequence intubation is preferable.
4. Video-laryngoscope is recommended (if available and provider is skilled in its use) to minimize distance between patient and intensivist, and reduce the transmission risk of COVID-19. ${ }^{18}$

5. Additionally, barrier devices (transparent intubation boxes or sheets) may be used while performing AGPs. However, use of these devices may make intubation difficult, prolonging the intubation time. Hence, caution should be exercised in hypoxemic patients.

\section{Mechanical Ventilation}

The true incidence of hypoxic respiratory failure in COVID-19 patients remains unknown. However, available data indicates that approximately $14 \%$ of COVID-19 patients develop severe disease needing oxygen administration, and approximately $5 \%$ require ICU admission and mechanical ventilation. ${ }^{18,19}$ High-flow nasal oxygen or NIPPV is used if conventional oxygen therapy fails to improve oxygenation in these patients with hypoxic respiratory failure. However, early intubation in a controlled setting is recommended over an emergency intubation. ${ }^{18,20}$

Hypoxia is deleterious and is linked to poor outcome in critically ill patients. ${ }^{21}$ Supplemental oxygen should be administered if peripheral oxygen saturation $\left(\mathrm{SpO}_{2}\right)$ falls below $92 \%$. $\mathrm{SpO}_{2}$ between 92 and 96\% is desirable for acutely ill medical patients. ${ }^{18}$ However, in patients of acute neuronal injury, hypoxemia and hypotension can worsen neurologic injury. In the absence of literature, oxygenation strategy based on cerebral oxygenation monitor (cerebral oximetry) may be considered to improve neurologic outcome.

Acute respiratory distress syndrome (ARDS) has been reported to develop in $42 \%$ of patients presenting with COVID-19 pneumonia, and 61 to $81 \%$ of those requiring ICU care. ${ }^{22}$ In patients of COVID-19 with ARDS requiring mechanical ventilation, a low tidal volume $(4-8 \mathrm{~mL} / \mathrm{kg}$ ) is recommended and plateau pressure (Pplat) $<30 \mathrm{~cm} \mathrm{H}_{2} \mathrm{O}$ should be targeted. ${ }^{18}$ In moderate to severe ARDS use of higher positive end-expiratory pressure (PEEP) is suggested over lower PEEP while monitoring for barotrauma and hemodynamic. However, in neurological patients with raised intracranial pressure (ICP), high PEEP or low tidal volume ventilation can cause hypercapnia, increase ICP, and worsen neurologic outcome. Hence, ICP monitoring is advisable for implementing this strategy.

\section{Prone Ventilation}

The COVID-19 patients with moderate-to-severe ARDS (i.e., $\mathrm{PaO}_{2} / \mathrm{FiO}_{2} \leq 200 \mathrm{~mm} \mathrm{Hg}$ ) and on mechanical ventilation, 12 to 16 hours of prone ventilation is recommended. ${ }^{18}$ Prone ventilation minimizes distension of ventral and collapse of dorsal alveoli, making ventilation more homogeneous. ${ }^{23}$ Recent meta-analyses have demonstrated a decrease in mortality with at least 12 hours of prone ventilation in patients with moderate-to-severe ARDS. ${ }^{24-27}$ In neurological patients, prone ventilation improves oxygenation but also increases ICP. ${ }^{28,29}$ Therefore in neurological patients with COVID-19, prone ventilation should be used with ICP monitoring and only when beneficial effects of prone ventilation outweigh 
potential harms from increase in ICP. In addition, neurological patients with absolute contraindications (unstable spine) should not receive prone ventilation. ${ }^{18}$

\section{Hemodynamic Management}

Neurological patients in the NCCU may develop hemodynamic instability or shock. The prevalence of shock in COVID-19 infection varies with severity of illness and may reach 20 to $35 \%$ in ICU patients. ${ }^{30,31}$ Cardiac enzymes are raised in 7 to $23 \%$ of patients with COVID-19. ${ }^{30-32}$ In approximately $40 \%$ of patients, shock from fulminant myocarditis results in mortality. ${ }^{33}$ Data in COVID-19 patients suggest that older age, associated comorbidities (diabetes and hypertension), reduced lymphocyte count, increased D-dimer level, and cardiac injury predispose to the development of shock. ${ }^{18,19,30,33}$

The advocated management of shock in COVID-19 patients is based on literature from non-COVID critically ill patients. Fluid responsiveness should be assessed using peripheral temperature, capillary refill time, and/or serum lactate levels. ${ }^{18}$ Since hypotension independently predicts mortality in neurocritical care patients, it should be aggressively treated using fluid resuscitation and vasopressor therapy. For initial resuscitation, balanced crystalloid solution is recommended. ${ }^{18}$ If unavailable, $0.9 \%$ saline is a reasonable alternative. ${ }^{18}$ For those not responding to fluid therapy, norepinephrine is the first-line vasoactive drug. ${ }^{18}$ Alternatively, vasopressin or epinephrine may be used. In COVID-19 patients with shock, mean arterial pressure between 60 and $65 \mathrm{~mm} \mathrm{Hg}$ should be targeted. In refractory shock, low-dose corticosteroid (intravenous hydrocortisone $200 \mathrm{mg} /$ day) is recommended ("shock-reversal" therapy). Advanced hemodynamic monitoring tools will be helpful in goal-directed therapy.

\section{Neurological Manifestations of COVID-19}

COVID-19-related neurological manifestations include stroke, headache, seizures, impaired consciousness, myalgia, and neuropathy (paresthesia and bowel/bladder dysfunction). . $^{34,35}$ COVID-19 patients have the likelihood of developing neurological problems such as critical illness myopathy and neuropathy, cerebrovascular disease, encephalopathy, and GBS. ${ }^{36,37}$ Distinguishing symptoms specific to COVID-19 from common complications of critical illness may be difficult.

Fever is common in NCCU and is associated with worse outcomes in patients with neurologic illness. Fever is mostly due to infection or underlying acute brain injury but COVID-19 can further complicate correct diagnosis. Fever should be managed utilizing both pharmacotherapy and device-related temperature therapies to reduce secondary brain injury from hyperthermia.

COVID-19 patients may have higher risk of developing seizures. ${ }^{38}$ Epileptiform abnormalities are detected in COVID-19 patients with encephalopathy. ${ }^{39}$ However, it is still unclear whether COVID-19 infection is directly responsible for seizures and if epileptiform activity affects outcome in these patients.

\section{Specific Neurological Conditions}

\section{Acute Ischemic Stroke}

Stroke is a frequent neurological manifestation in COVID-19 patients and they may develop AIS despite anticoagulant chemoprophylaxis. Data of COVID patients from China $^{35,40}$ showed that AIS occurred in the elderly or middle aged with known vascular risk factors. Whereas COVID-19 AIS patients from New York were younger, and had more severe strokes (predominantly cryptogenic variety), higher D-dimer levels, and increased mortality vis-a-vis non-COVID strokes. ${ }^{41}$ Comorbidities (diabetes, hypertension, obesity, and cardiovascular disease) increase the risk of stroke in COVID-19 patients.

Recent literature suggests stroke in COVID-19 to be either due to endothelitis ${ }^{42}$ from direct viral infection via angiotensin-converting enzyme 2 (ACE2) receptors (hence the possible use of anticytokine drugs [tocilizumab, sarilumab], ${ }^{43,44}$ statins, ${ }^{45-47}$ and anti-inflammatory drugs ${ }^{48,49}$ ) and/or through COVID-19-associated coagulopathy ${ }^{50}$ reflected by increased fibrinogen and D-dimer (consequently, low molecular weight heparin [LMWH] or unfractionated heparin [UFH] is used for thromboprophylaxis).

In COVID-19 era, a predefined protocol for stroke management reduces the risk to HCPs and improves delivery of patient care. On hospital arrival, stroke patients should be screened for clinical symptoms of SARI (cough and fever $>38^{\circ} \mathrm{C}$ ), headache, dyspnea/tachypnea (respiratory rate $>24 / \mathrm{min}$ ), chest pain, myalgia, vomiting, and diarrhea, contact with COVID-19 patient, international travel in the preceding 2 weeks, and residence in high-risk/containment zone. If the above are positive or cannot be ascertained, "Protected Code Stroke" (PCS) should be activated ${ }^{51}$ which includes the following:

(1) Evaluation of the patient (wearing surgical mask) simultaneously by neurologist or member of stroke/ intervention team and anesthesiologist/intensivist (using appropriate PPE).

(2) If there is a high demand for oxygen $\left(\mathrm{FiO}_{2}>0.5\right)$ or decreased consciousness, elective intubation (with recommended precautions) should be done, before shifting to radiology or interventional neuroradiology (INR) suite. Choices of drugs include ketamine or etomidate to maintain blood pressure (BP). Any decline in BP should prompt early vasopressor use.

Emergency management and referral of AIS patients in COVID-19 pandemic:

1. Covid-19 designated hospital with stroke facility: If the patient is asymptomatic, negative on screening, and is not from a hot spot/containment zone, the hospital's "codestroke" pathway may be followed. For COVID-19 suspect, emergency services should not be delayed for lack of test. However, swab should be sent for testing and PCS should be activated.

2. Non-COVID hospital with stroke facility: If COVID-19 is suspected, PCS should be activated. Patient should be stabilized (including thrombolysis if within time window) 
and referred to the nearest COVID-19 designated hospital with stroke unit.

3. Non-COVID hospital without stroke facility: Suspected COVID-19 AIS patient should be immediately referred to the nearest COVID-19 designated hospital with stroke unit.

Imaging in COVID-19: There should be a predesignated computed tomography (CT) and INR suite for COVID-19 suspect/confirmed patients (Stroke Green Pathway). ${ }^{52}$ If this is not possible, disinfection of the suite is performed between the cases. CT suite should be prenotified before shifting of the patient to allow for preparation. The preferred imaging is noncontrast $\mathrm{CT}^{53}$ of brain within 20 minutes of arrival with follow-up CT scan repeated after 24 hours. All patients with suspected/confirmed COVID-19 should additionally undergo CT imaging of the lungs as typical lesions (subsegmental ground glass opacities) are seen in up to $82 \%$ of cases.

Thrombectomy in INR suite may either be performed under general anesthesia (GA) or monitored anesthesia care (MAC) and the decision should be individualized based on patient condition. ${ }^{54}$ GA should be administered to those already intubated, those with active cough/vomiting, posterior circulation or dominant hemisphere stroke, severe stroke, Glasgow Coma Scale (GCS) score < 9, and agitated/ aphasic/uncooperative patient. MAC should be provided to others. For patients undergoing thrombectomy with MAC, oxygen should be provided with nasal prongs below the surgical mask and capnography must be monitored continuously. No or minimal sedation should be used to minimize rescue airway maneuvers. For urgent conversion from MAC to GA, airborne precautions should be followed, intubation should be performed by an experienced anesthesiologist using video-laryngoscope, and systolic BP should be maintained > $140 \mathrm{~mm} \mathrm{Hg}$. Extubation should be done in NPR; however, if performed in INR suite, all precautions should be followed.Postprocedure care: Dyna or cone-beam head CT imaging should be done in INR suite to avoid transfer to CT room. In the NCCU, suspected/confirmed COVID-19 patient should be cared in NPR, if available. If trachea is not extubated, the patient should be sedated and ventilated in the NCCU with sedation breaks every 8 hours for neurological examination. Invasive BP monitoring should preferably be done. Early extubation should be considered to optimize scarce NCCU resources.

\section{Traumatic Brain Injury}

With increasing numbers of COVID-19 patients, changes in admission policies are required to optimally utilize NCCU beds and manpower for both COVID-19 and non-COVID patients. TBI patients with minor lesions (undisplaced fractures or traumatic SAH with GCS of 15) may not require NCCU admission. ${ }^{55}$

Once NCCU receives information regarding arrival of TBI patient, "Protected Code Brain" should be activated including protective measures against COVID-19 infection, mentioned earlier. NCCU management of TBI should follow existing norms of care. ${ }^{56}$ Morbidity and mortality in COVID-19 patients could be from end-organ involvement which can worsen in presence of severe TBI.

\section{Acute Spinal Cord Injury}

Respiratory dysfunction leading to increased risk of pulmonary infection is the main reason for complications and death in SCI. SCI-induced respiratory dysfunction further increases the patient's susceptibility to COVID-19 pneumonia. Patients of SCI may not present with typical symptoms of COVID-19 due to altered physiology (such as temperature dysregulation, impaired cough, and abnormal sensations below the neurological level of injury) which may pose a unique diagnostic challenge during the current pandemic. Given an already reduced lung capacity and impaired cough, a high level of suspicion for respiratory illness is needed to ensure an early diagnosis of COVID-19 infection in this population. ${ }^{57}$

All patients with SCI should be screened for COVID-19 at admission and treated as per the standard protocols of acute SCI. Appropriate precautions against the disease including use of PPE should be undertaken especially during assisted coughing to avoid exposure to aerosolized secretions. ${ }^{58-61}$

\section{Subarachnoid Hemorrhage}

aSAH is a neurologic emergency and carries significant morbidity and mortality. The neuroanesthesiologist and/or neurointensivist may be involved in managing these patients during resuscitation (poor grade), diagnostic cerebral angiography, aneurysm clipping or endovascular coiling, postoperative care in the NCCU, transportation to/from imaging suite, and vasospasm management in the INR suite.

Following aSAH, there is heightened sympathetic drive and inflammation resulting in heart and lung dysfunction. This can manifest as breathlessness, oxygen desaturation, fever, and bilateral lung infiltrates due to neurogenic pulmonary edema. These features could mimic COVID-19 infection. Misdiagnosing potential COVID-19 lung manifestations for aSAH-related pulmonary complications can predispose HCPs to COVID-19 infection. There are isolated reports of SAH following COVID-19 infection ${ }^{62}$ though; it is difficult to say if they are causal or coincidental. ${ }^{63}$ Published data shows increased postoperative mortality in COVID-19 infected patients. ${ }^{64}$ Based on history and clinical features, COVID-19 infection should be suspected. However, SAH management (clipping or coiling) should not wait till laboratory confirmation of COVID-19 infection.

COVID-19 affects the endothelial/epithelial ACE2 receptors. It is currently unclear whether this increases the possibility of vasospasm/thrombosis leading to delayed cerebral ischemia. Vasospasm management after aSAH often requires intra-arterial administration of nimodipine or milrinone. Transporting COVID-19 patient repeatedly from NCCU to INR suite for intra-arterial therapy may be challenging with regards to sanitization of the pathway and extra care required during transport of such patients. In such situations, to minimize transfers, intravenous milrinone or nimodipine may be more suitable. When necessary, angiography may be considered in discussion with the INR team. 
In the NCCU, respiratory failure and hypoxemia can accelerate cerebral ischemia. Where available, ventilation and oxygenation can be titrated based on cerebral oxygenation monitoring.

\section{Status Epilepticus}

Focal status epilepticus (SE) has been reported as an initial manifestation of COVID-19 infection. ${ }^{65}$ There are no other similar reports. However, caution should be exercised in patients presenting with SE requiring NCCU admission.

\section{Neuromuscular Disorders}

Neuromuscular complications may be encountered during the pandemic. COVID-19 presents as myalgia in 44 to $70 \%$ of patients. $^{31,66,}$ Patients with motor neuron disease who have underlying respiratory muscle weakness and immunocompromised patients are at increased risk of contracting COVID-19.31,66 Patients with neuromuscular disorders (NMDs) receive immunosuppressant and immunomodulating therapy, thereby increasing their susceptibility. Therefore, patients with neuromuscular weakness of new-onset or from underlying known disorder should be evaluated for COVID-19 during this pandemic.

Viral infections with Epstein-Barr virus, H1N1, influenza, and Zika virus are associated with development of GBS. GBS is also reported with other corona virus infections. ${ }^{67,68}$ Though GBS has been reported with COVID-19, causality is uncertain. ${ }^{69}$

Pulmonary infection is one of the triggers for myasthenia crisis leading to NCCU admission. Clinical suspicion and laboratory testing for COVID-19 is essential during this pandemic. Hydroxychloroquine and chloroquine, which are used for prophylaxis and/or treatment of COVID-19, may cause exacerbation of MG. ${ }^{70-72}$ Hydroxychloroquine is not recommended in patients with Duchenne or Becker muscular dystrophy. ${ }^{70}$

Close monitoring for worsening of underlying disease or respiratory function is recommended in moderate-to-high risk patients with NMD. Currently, no NMD-specific recommendations are available for COVID-19 patients. Use of corticosteroids and other immunosuppressants is based on clinical status of COVID-19 patient and seriousness of underlying NMD.

\section{Postoperative Neurosurgical Patients}

Patients with brain tumors receive steroids to reduce cerebral edema which can lead to immune suppression. Although few experts suggest using steroids during early phase of COVID-19 infection, consensus is lacking. Immune-suppressed neurosurgical patients have increased risk of developing severe COVID-19 infection in the perioperative period requiring NCCU care. Hence, judicious decisions are needed regarding dose and duration of steroid therapy. Studies have reported increased postoperative pulmonary complications and higher mortality following surgery in COVID-19 patients. ${ }^{64,73,74}$ This should be considered during postoperative care in the NCCU.

\section{Nonneurological Issues and Their Management in NCCU}

\section{Thromboprophylaxis in COVID-19}

Observational data have suggested increased thrombotic complications in COVID-19, particularly in those with
ARDS. ${ }^{75-79}$ As a standard-of-care, nonambulant COVID-19 patients in NCCU with respiratory failure or coexisting cancer, diabetes mellitus, hypertension, or heart failure, should receive prophylactic anticoagulation with daily LMWHs or twice-daily subcutaneous UFH, unless contraindicated..$^{80-82}$ Intermittent pneumatic compression should be considered when pharmacological prophylaxis is contraindicated.

The role of thromboprophylaxis for asymptomatic COVID-19 patients or those with mild symptoms, but significant comorbidities, is uncertain. Since supporting data are lacking, pharmacological prophylaxis should be restricted to high-risk patients (bedridden, prior thromboembolism, and/ or active malignancy). ${ }^{83}$

There are no data to demonstrate improved clinical outcomes with high-dose anticoagulation. ${ }^{83}$ The most important consideration during anticoagulation is risk of bleeding, particularly, ICH in postoperative patients and aggravation of bleed in SAH patients. COVID-19 patients with high risk for thrombosis also have increased bleeding possibility. ${ }^{82}$ Therefore, anticoagulation should be administered after understanding the risk of $\mathrm{ICH}^{84}$

\section{Nutrition}

Frailty and malnourishment may contribute to increased morbidity and mortality in COVID-19. Early nutritional assessment and institution of appropriate feeds (preferably enteral) is vital. ${ }^{85}$ Prone position, which is often used in COVID-19 patients, does not contraindicate enteral nutrition. ${ }^{86}$ Dysphagia is common after neurological injury such as stroke and muscle weakness from critical illness. Therefore, careful assessment of swallowing after decannulation or extubation is needed, followed by gradual feeding.

\section{Tracheostomy}

Where possible, an early extubation is desirable. However, coughing and open T-piece weaning, which generate aerosols, should be avoided. Drugs such as dexmedetomidine facilitate smooth extubation without significant cardiorespiratory system activation. Extubation can be performed directly from low pressure support ventilation if the patient fulfills extubation criteria. Tracheostomy in NCCU is indicated when extubation is not possible or ventilator weaning trials fail. Mortality in ventilated patients with COVID-19 pneumonitis is approximately 50\%. ${ }^{87}$ Literature suggests that acute lung injury phase of COVID-19 pneumonitis lasts for approximately 10 to 14 days. ${ }^{87}$ Viral load is likely to be low after 3 weeks of first symptoms. Since tracheostomy is AGP with high risk for infection transmission to HCPs, it is recommended to delay tracheostomy if possible in COVID-19 patients. ${ }^{87}$ However, some COVID-19 neurological patients may require early tracheostomy such as those predicted to fail primary extubation (lower cranial involvement, poor GCS score, severe stroke, difficult airway, high cervical SCI). There is however no guideline to inform timing of tracheostomy in COVID-19 patients. Decision about timing must balance the risks of prolonged intubation for the patient against risks to the HCPs involved in tracheostomy. ${ }^{87,88}$ 
Both percutaneous and surgical tracheostomy can be performed in COVID-19 neurological patients. The procedure should be performed with recommended precautions in a NPR with closed suction and minimum staff. ${ }^{87,88}$ Use of fiberoptic scope during percutaneous tracheostomy is not recommended in COVID-19 patients ${ }^{87}$ Preoxygenation (100\% oxygen for 5 minutes) before the procedure is recommended. Aerosol generation can be minimized by the following:

- Prevent breach of ETT cuff during tracheostomy.

- Clamp ETT before disconnecting the circuit.

- Reduce ventilator pressure and/or frequency when trachea is punctured, dilated, or opened surgically (consider suspending ventilation if patient condition allows) and resume ventilation once cuff is inflated.

- Prefer surgical ties to diathermy to reduce vapor plumes. ${ }^{87}$

- In tracheostomized patients, triple layer surgical mask should be applied over the face to minimize spread of COVID-19 infection. ${ }^{87}$

\section{Extracorporeal Membrane Oxygenation}

At present, data are insufficient to recommend extracorporeal membrane oxygenation (ECMO) in COVID-19 patients with refractory hypoxemia. ${ }^{20}$ In mechanically ventilated COVID-19 patients with severe ARDS and refractory hypoxemia notwithstanding various treatments to optimize ventilation and improve oxygenation, veno-venous ECMO may be considered in carefully selected patients. ${ }^{18}$ However, due to high cost, limited availability, and need for trained staff and infrastructure, it remains an extremely limited resource-intense technique. At present, ECMO should be restricted to carefully chosen patients. Studies reporting outcomes in COVID-19 patients receiving ECMO will guide future practice. ${ }^{18,20,89}$

\section{Cardiopulmonary Resuscitation}

CPR is a life-saving procedure. However, it poses risks of COVID-19 transmission to the administering HCPs. ${ }^{90}$ American Heart Association has issued the interim guidance on CPR for the HCPs during COVID-19 outbreak. ${ }^{91}$ The decision to perform CPR during pandemic should be based on likely neurological outcome of the patients. In patients with poor outcome, family should be counseled about prognosis, goals of care, and do-not-resuscitate status. To protect the CPR team, resuscitation should be performed only after the response team has donned appropriate PPE.

\section{Targeted Temperature Management during COVID-19 Pandemic}

Targeted temperature management (TTM) is an important component of acute care of unresponsive postcardiac arrest patients. There is no literature regarding the practice of TTM during COVID pandemic. Best practices as per existing guidelines and discussions with family regarding outcome should guide its use.

\section{Communication and Psychological Aspects in NCCU}

Most patients in NCCU are critically ill. Constant communication with the family is often required to update them about the patient status and for consent for procedures and care. However, if family members are also COVID-19 positive, they are likely to be at home or institutional quarantine making communication and shared decision-making difficult.

Patients with positive COVID-19 status are likely to be stressed about the prognosis. This can affect systemic physiology, and in turn, neurological outcomes. Children and postoperative patients are more vulnerable to psychological issues if parents or family members are unable to visit them in the NCCU due to quarantine or their own hospitalization. Patients dying in adjacent beds in the NCCU can adversely affect patients and may even develop new-onset neuropsychiatric manifestations. Stress is also likely among HCPs caring for COVID-19 patients in the NCCU. Early recognition, prompt psychological support, and rotation in postings are crucial for restoring HCP's wellness.

\section{COVID-19 Retesting in NCCU}

Neurological COVID-19 patients are likely to stay in the NCCU for a long time. Repeat testing for COVID-19 helps optimize resources and plan patient care. There is no clarity on the timing and frequency of retesting in NCCU. It should be performed as per local guidelines.

\section{Conclusion}

Management of critically ill neurological patients during COVID19 pandemic is challenging and requires a good understanding of pathophysiology and management aspects of COVID-19 along with that of the primary neurological condition. Strategies to streamline workflows, robust infection control measures, and ensuring safety of HCPs are crucial in the NCCU management of critically ill neurological patients during this pandemic.

\section{Conflict of Interest}

G.P.R. presently serves as a Director-at-Large of the Society for Neuroscience in Anesthesiology and Critical Care (SNACC) Board of Directors. Other authors have no conflict of interest to declare.

\section{References}

1 Tobin MJ, Laghi F, Jubran A. Why COVID-19 silent hypoxemia is baffling to physicians. Am J Respir Crit Care Med 2020. Doi:10.1164/rccm.202006-2157CP

2 Xiao AT, Tong YX, Zhang S. False-negative of RT-PCR and prolonged nucleic acid conversion in COVID-19: rather than recurrence. J Med Virol 2020. Doi: 10.1002/jmv.25855

3 Goh KJ, Wong J, Tien JC, et al. Preparing your intensive care unit for the COVID-19 pandemic: practical considerations and strategies. Crit Care 2020;24(1):215

4 Martland AM, Huffines M, Henry K. Surge priority planning COVID-19: critical care staffing and nursing considerations. CHEST 2020. Available at: http://www.chestnet. org/Guidelines-and-Resources/Resources/Surge-PriorityPlanning-COVID-19-Critical-Care-Staffing-and-NursingConsiderations. Accessed June 10, 2020 
5 New WHO tools launched to help hospitals manage surge in COVID-19 patients. April 8, 2020. Available at: https://www. euro.who.int/en/health-topics/health-emergencies/coronavirus-covid-19/news/news/2020/4/new-who-tools-launchedto-help-hospitals-manage-surge-in-covid-19-patients. Accessed June 10, 2020

6 List N. Disinfectants for Use Against SARS-CoV-2 (COVID-19). 2020. Available at: https://www.epa.gov/pesticide-registration/list-n-disinfectants-use-against-sars-cov-2. Accessed June 15,2020

7 COVID-19. Guidelines on disinfection of common public places including offices. 2020. Available at: https://www.mohfw. gov.in/pdf/Guidelinesondisinfectionofcommonpublicplaces includingoffices.pdf. Accessed June 15, 2020

8 Instructions to all major and minor ports for dealing with novel coronavirus (COVID-19) pandemic. Cleaning agents and disinfectants. 2020. Available at: https://www.mohfw.gov.in/ pdf/DGSOrder04of2020.pdf. Accessed June 15, 2020

9 Coronavirus Disease. 2019 (COVID-19): Standard Operating Procedure (SOP) for transporting a suspectirmed case of COVID-19. 2020. Available at: https://www.mohfw.gov.in/pdf/ StandardOperatingProcedureSOPfortransportingasuspector confirmedcaseofCOVID19.pdf. Accessed June 10, 2020

10 Guidelines for disinfection of quarantine facility (for COVID19). 2020. Available at: https://ncdc.gov.in/WriteReadData/ 1892s/89168637271584172711.pdf. Accessed June 15, 2020

11 Novel Coronavirus Disease. 2019 (COVID-19): Guidelines on rational use of Personal Protective Equipment. 2020. Available at: https://www.mohfw.gov.in/pdf/GuideliOnes onrationaluseofPersonalProtectiveEquipment.pdf. Accessed June 10, 2020

12 Novel Coronavirus Disease. 2019(COVID-19): Additional guidelines on rational use of Personal Protective Equipment (setting approach for Health functionaries working in non-COVID areas). 2020. Available at: https://www.mohfw.gov.in/pdf/Additional guidelinesonrationaluseofPersonalProtectiveEquipmentsettingapproachforHealthfunctionariesworkinginnon COVIDareas.pdf. Accessed June 10, 2020

13 National guidelines for infection prevention and control in healthcare facilities. 2020. Available at: https://www.mohfw. gov.in/pdf/National\%20Guidelines\%20for\%20IPC\%20in\%20 HCF\%20-\%20final\%281\%29.pdf. Accessed June 10, 2020

14 Using Personal Protective Equipment (PPE). 2020. Available at: https://www.cdc.gov/coronavirus/2019-ncov/hcp/using-ppe. html. Accessed June 10, 2020

15 Steps to put on personal protective equipment (PPE). 2020. Available at: https://www.who.int/csr/disease/ebola/put_on_ ppequipment.pdf. Accessed June 10, 2020

16 El-Boghdadly K, Wong DJN, Owen R, et al. Risks to healthcare workers following tracheal intubation of patients with COVID-19: a prospective international multicentre cohort study. Anaesthesia 2020. Doi: 10.1111/anae.15170

17 World Health Organization. Clinical management of severe acute respiratory infection (SARI) when COVID-19 disease is suspected: Interim guidance. March 13, 2020. Available at: https://www.who.int/docs/default-source/coronaviruse/ clinical-management-of-novel-cov.pdf?sfvrsn=bc7da517_2. Accessed June 16, 2020

18 Alhazzani W, Møller MH, Arabi YM, et al. Surviving Sepsis Campaign: guidelines on the management of critically ill adults with Coronavirus Disease 2019 (COVID-19) Intensive Care Med 2020;46(5):854-887

19 Wu Z, McGoogan JM. Characteristics of and important lessons from the coronavirus disease 2019 (COVID-19) outbreak in China: summary of a report of 72314 cases from the Chinese Center for Disease Control and Prevention. JAMA 2020. Doi: 10.1001/jama.2020.2648
20 NIH-Coronavirus Disease. 2019 (COVID-19) Treatment Guideline. 2020. Available at: https://files.covid19treatment guidelines.nih.gov/guidelines/covid19treatmentguidelines. pdf. Accessed June 16, 2020

21 van den Boom W, Hoy M, Sankaran J, et al. The search for optimal oxygen saturation targets in critically ill patients: observational data from large ICU databases. Chest 2020;157(3):566-573

22 Wu C, Chen X, Cai Y, et al. Risk factors associated with acute respiratory distress syndrome and death in patients with coronavirus disease 2019 pneumonia in Wuhan, China. JAMA Intern Med 2020. Doi: 10.1001/jamainternmed.2020.0994

23 Cornejo RA, Díaz JC, Tobar EA, et al. Effects of prone positioning on lung protection in patients with acute respiratory distress syndrome. Am J Respir Crit Care Med 2013;188(4):440-448

24 Munshi L, Del Sorbo L, Adhikari NKJ, et al. Prone position for acute respiratory distress syndrome. A systematic review and meta-analysis. Ann Am Thorac Soc 2017;14(Supplement_4):S280-S288

25 Bloomfield R, Noble DW, Sudlow A. Prone position for acute respiratory failure in adults. Cochrane Database Syst Rev 2015; ( 11):CD008095

26 Mora-Arteaga JA, Bernal-Ramírez OJ, Rodríguez SJ. The effects of prone position ventilation in patients with acute respiratory distress syndrome. A systematic review and meta-analysis. Med Intensiva 2015;39(6):359-372

27 Lee JM, Bae W, Lee YJ, Cho YJ. The efficacy and safety of prone positional ventilation in acute respiratory distress syndrome: updated study-level meta-analysis of 11 randomized controlled trials. Crit Care Med 2014;42(5):1252-1262

28 Roth C, Ferbert A, Deinsberger W, et al. Does prone positioning increase intracranial pressure? A retrospective analysis of patients with acute brain injury and acute respiratory failure. Neurocrit Care 2014;21(2):186-191

29 Nekludov M, Bellander BM, Mure M. Oxygenation and cerebral perfusion pressure improved in the prone position. Acta Anaesthesiol Scand 2006;50(8):932-936

30 Yang X, Yu Y, Xu J, et al. Clinical course and outcomes of critically ill patients with SARS-CoV-2 pneumonia in Wuhan, China: a single-centered, retrospective, observational study. Lancet Respir Med 2020;8(5):475-481

31 Wang D, Hu B, Hu C, et al. Clinical characteristics of 138 hospitalized patients with 2019 novel coronavirus-infected pneumonia in Wuhan, China. JAMA 2020;323:1061-1069

32 Zhou F, Yu T, Du R, et al. Clinical course and risk factors for mortality of adult inpatients with COVID-19 in Wuhan, China: a retrospective cohort study. Lancet 2020;395(10229):1054-1062

33 Ruan Q, Yang K, Wang W, Jiang L, Song J. Clinical predictors of mortality due to COVID-19 based on an analysis of data of 150 patients from Wuhan, China. Intensive Care Med 2020;46(5):846-848

34 Guan WJ, Ni ZY, Hu Y, et al; China Medical Treatment Expert Group for Covid-19. Clinical characteristics of coronavirus disease 2019 in China. N Engl J Med 2020;382(18): $1708-1720$

35 Mao L, Jin H, Wang M, et al. Neurologic manifestations of hospitalized patients with coronavirus disease 2019 in Wuhan, China. JAMA Neurol 2020;e201127. Doi: 10.1001/jamaneurol.2020.1127

36 Oxley TJ, Mocco J, Majidi S, et al. Large-vessel stroke as a presenting feature of Covid-19 in the young. N Engl J Med 2020;382(20):e60

37 Filatov A, Sharma P, Hindi F, Espinosa PS. Neurological complications of coronavirus disease (COVID-19): encephalopathy. Cureus 2020;12(3):e7352

38 Hepburn M, Mullaguri N, George P, et al. Acute symptomatic seizures in critically ill patients with COVID-19: is there an association? Neurocrit Care 2020. Doi: 10.1007/ s12028-020-01006-1 
39 Louis S, Dhawan A, Newey C, et al. Continuous electroencephalography (cEEG) characteristics and acute symptomatic seizures in COVID-19 patients. medRxiv; 2020. Doi: 10.1101/2020.05.26.20114033

40 Li Y, Wang M, Zhou Y, et al. Acute cerebrovascular disease following covid-19: a single center, retrospective, observational study. Lancet 2020. Doi: 10.2139/ssrn.3550025

41 Yaghi S, Ishida K, Torres J, et al. SARS-CoV-2 and stroke in a New York healthcare system. Stroke 2020;51(7):2002-2011

42 Varga Z, Flammer AJ, Steiger P, et al. Endothelial cell infection and endotheliitis in COVID-19. Lancet 2020;395(10234): 1417-1418

43 Michot JM, Albiges L, Chaput N, et al. Tocilizumab, an anti-IL-6 receptor antibody, to treat COVID-19-related respiratory failure: a case report. Ann Oncol 2020;31(7):961-964

44 Monteleone G, Sarzi-Puttini PC, Ardizzone S. Preventing COVID-19-induced pneumonia with anticytokine therapy. Lancet Rheumatol 2020;2(5):e255-e256

45 Castiglione V, Chiriacò M, Emdin M, Taddei S, Vergaro G. Statin therapy in COVID-19 infection. Eur Heart J Cardiovasc Pharmacother 2020;42

46 Dashti-Khavidaki S, Khalili H. Considerations for statin therapy in patients with COVID-19. Pharmacotherapy 2020;40(5):484-486

47 Virani SS. Is There a Role For Statin Therapy in Acute Viral Infections? Cardiology Magazine; 2020. Available at: https:// www.acc.org/latest-in-cardiology/articles/2020/03/18/15/09/ is-there-a-role-for-statin-therapy-in-acute-viral-infectionscovid-19. Accessed July 9, 2020

48 Zhang W, Zhao Y, Zhang F, et al. The use of anti-inflammatory drugs in the treatment of people with severe coronavirus disease 2019 (COVID-19): the perspectives of clinical immunologists from China. Clin Immunol 2020;214:108393

49 Stebbing J, Phelan A, Griffin I, et al. COVID-19: combining antiviral and anti-inflammatory treatments. Lancet Infect Dis 2020;20(4):400-402

50 Connors JM, Levy JH. COVID-19 and its implications for thrombosis and anticoagulation. Blood 2020;135(23):2033-2040

51 Khosravani H, Rajendram P, Notario L, Chapman MG, Menon BK. Protected code stroke: hyperacute stroke management during the coronavirus disease 2019 (COVID-19) pandemic. Stroke 2020;51(6):1891-1895

52 Neurointervention Committee of the Chinese Medical Doctor Association. Recommendation of experts on prevention and control of new coronavirus infection in neurointerventional specialty (version 1) [D] Chin J Cerebrovasc Dis 2020;17:107-112

53 Powers WJ, Rabinstein AA, Ackerson T, et al. Guidelines for the early management of patients with acute ischemic stroke: 2019 update to the 2018 guidelines for the early management of acute ischemic stroke. A guideline for healthcare professionals from the American Heart Association/American Stroke Association. Stroke 2019;50(12):e344-e418

54 Sharma D, Rasmussen M, Han R, et al. Anesthetic management of endovascular treatment of acute ischemic stroke during COVID-19 pandemic: consensus statement from Society for Neuroscience in Anesthesiology \& Critical Care (SNACC): endorsed by Society of Vascular \& Interventional Neurology (SVIN), Society of Neurolnterventional Surgery (SNIS), Neurocritical Care Society (NCS), European Society of Minimally Invasive Neurological Therapy (ESMINT) and American Association of Neurological Surgeons (AANS) and Congress of Neurological Surgeons (CNS) Cerebrovascular Section. J Neurosurg Anesthesiol 2020;32(3):193-201

55 COVID-19: Statement Regarding the Management of Minimal, Mild and Moderate Head Injury. 2020. Available at: http:// media.neurotrauma.nu/2020/03/SNC-statement-COVID-19. pdf. Accessed June 15, 2020
56 Carney N, Totten AM, O'Reilly C, et al. Guidelines for the management of severe traumatic brain injury, fourth edition. Neurosurgery; 2017;80:6-15

57 Korupolu R, Stampas A, Gibbons C, Hernandez Jimenez I, Skelton F, Verduzco-Gutierrez M. COVID-19: screening and triage challenges in people with disability due to Spinal Cord Injury. Spinal Cord Ser Cases 2020;6(1):35

58 Dicks MA, Clements ND, Gibbons CR, Verduzco-Gutierrez M, Trbovich M. Atypical presentation of Covid-19 in persons with spinal cord injury. Spinal Cord Ser Cases 2020;6(1):38

59 Rodríguez-Cola M, Jiménez-Velasco I, Gutiérrez-Henares F, et al. Clinical features of coronavirus disease 2019 (COVID-19) in a cohort of patients with disability due to spinal cord injury. Spinal Cord Ser Cases 2020;6(1):39

60 Stillman MD, Capron M, Alexander M, Di Giusto ML, Scivoletto G. COVID-19 and spinal cord injury and disease: results of an international survey. Spinal Cord Ser Cases 2020;6(1):21

61 Basics guidance on management of spinal cord injury patients during coronavirus (COVID-19) pandemic. 2020. Available at: https://www.iscos.org.uk/uploads/CV-19/updated\%20files\% 204\%2024\%2020/ENG_Basic_guidance_on_management.pdf. Accessed June 16, 2020

62 Craen A, Logan G, Ganti L. Novel coronavirus disease 2019 and subarachnoid hemorrhage: a case report. Cureus 2020;12(4):e7846

63 Sharifi-Razavi A, Karimi N, Rouhani N. COVID-19 and intracerebral haemorrhage: causative or coincidental? New Microbes New Infect 2020;35:100669

64 COVIDSurg Collaborative. Mortality and pulmonary complications in patients undergoing surgery with perioperative SARSCoV-2 infection: an international cohort study. Lancet 2020. Doi: 10.1016/S0140-6736(20)31182-X

65 Vollono C, Rollo E, Romozzi M, et al. Focal status epilepticus as unique clinical feature of COVID-19: a case report. Seizure 2020;78:109-112

66 Huang C, Wang Y, Li X, et al. Clinical features of patients infected with 2019 novel coronavirus in Wuhan, China. Lancet 2020;395(10223):497-506

67 Kim JE, Heo JH, Kim HO, et al. Neurological complications during treatment of Middle East respiratory syndrome. J Clin Neurol 2017;13(3):227-233

68 Sharma K, Tengsupakul S, Sanchez O, Phaltas R, Maertens P. Guillain-Barré syndrome with unilateral peripheral facial and bulbar palsy in a child: a case report. SAGE Open Med Case Rep 2019;7:X19838750

69 Zhao H, Shen D, Zhou H, Liu J, Chen S. Guillain-Barré syndrome associated with SARS-CoV-2 infection: causality or coincidence? Lancet Neurol 2020;19(5):383-384

70 Elavarasi A, Goyal V. Hydroxychloroquine and myasthenia gravis-can one take this risk? Ann Indian Acad Neurol 2020;23:360-361

71 Varan O, Kucuk H, Tufan A. Myasthenia gravis due to hydroxychloroquine. Reumatismo 2015;67(3):849

72 Klimek A. The myasthenic syndrome after chloroquine [in Polish]. Neurol Neurochir Pol 1999;33(4):951-954

73 Myles PS, Maswime S. Mitigating the risks of surgery during the COVID-19 pandemic. Lancet 2020;396(10243):2-3

74 Aminian A, Safari S, Razeghian-Jahromi A, Ghorbani M, Delaney CP. COVID-19 outbreak and surgical practice: unexpected fatality in perioperative period. Ann Surg 2020;272(1):e27-e29

75 Cui S, Chen S, Li X, Liu S, Wang F. Prevalence of venous thromboembolism in patients with severe novel coronavirus pneumonia. J Thromb Haemost 2020;18(6):1421-1424

76 Dolhnikoff M, Duarte-Neto AN, de Almeida Monteiro RA, et al. Pathological evidence of pulmonary thrombotic phenomena in severe COVID-19. J Thromb Haemost 2020;18(6):1517-1519 
77 Goyal P, Choi JJ, Pinheiro LC, et al. Clinical characteristics of Covid-19 in New York City. N Engl J Med 2020;382(24): 2372-2374

78 Klok FA, Kruip MJHA, van der Meer NJM, et al. Confirmation of the high cumulative incidence of thrombotic complications in critically ill ICU patients with COVID-19: an updated analysis. Thromb Res 2020;191:148-150

79 Lodigiani C, Iapichino G, Carenzo L, et al; Humanitas COVID-19 Task Force. Venous and arterial thromboembolic complications in COVID-19 patients admitted to an academic hospital in Milan, Italy. Thromb Res 2020;191:9-14

80 Poissy J, Goutay J, Caplan M, et al; Lille ICU Haemostasis COVID-19 group. Pulmonary embolism in COVID-19 patients: awareness of an increased prevalence. Circulation 2020. Doi:10.1161/CIRCULATIONAHA.120.047430

81 Tang N, Bai H, Chen X, Gong J, Li D, Sun Z. Anticoagulant treatment is associated with decreased mortality in severe coronavirus disease 2019 patients with coagulopathy. J Thromb Haemost 2020;18(5):1094-1099

82 Wang T, Chen R, Liu C, et al. Attention should be paid to venous thromboembolism prophylaxis in the management of COVID-19. Lancet Haematol 2020;7(5):e362-e363

83 World Health Organization. Clinical management of severe acute respiratory infection when novel coronavirus (2019-nCoV) infection is suspected: interim guidance. World Health Organization; 2020. Available at: https://apps.who.int/ iris/handle/10665/330893. Accessed January 28, 2020

84 Dogra S, Jain R, Cao M, et al. Hemorrhagic stroke and anticoagulation in COVID-19. J Stroke Cerebrovasc Dis 2020. Doi:10.1016/j.jstrokecerebrovasdis.2020.104984
85 Barazzoni R, Bischoff SC, Breda J, et al; endorsed by the ESPEN Council. ESPEN expert statements and practical guidance for nutritional management of individuals with SARS-CoV-2 infection. Clin Nutr 2020;39(6):1631-1638

86 Saez de la Fuente I, Saez de la Fuente J, Quintana Estelles $M D$, et al. Enteral nutrition in patients receiving mechanical ventilation in a prone position. J Parenter Enteral Nutr 2016;40(2):250-255

87 National Tracheostomy Safety Project. Considerations for tracheostomy in the COVID-19 outbreak. 2020. Available at: https://icmanaesthesiacovid-19.org/considerations-for-tracheostomy-in-the-covid-19-outbreak. Accessed June 13, 2020

88 Shiba T, Ghazizadeh S, Chhetri D, St John M, Long J. Tracheostomy considerations during the COVID-19 pandemic. OTO Open 2020;4(2):X20922528

89 MacLaren G, Fisher D, Brodie D. Preparing for the most critically ill patients with COVID-19: the potential role of extracorporeal membrane oxygenation. JAMA 2020. Doi: 10.1001/ jama.2020.2342

90 Andersen LW, Holmberg MJ, Berg KM, Donnino MW, Granfeldt A. In-hospital cardiac arrest: a review. JAMA 2019;321(12): $1200-1210$

91 Edelson DP, Sasson C, Chan PS, et al; American Heart Association ECC Interim COVID Guidance Authors. Interim guidance for basic and advanced life support in adults, children, and neonates with suspected or confirmed COVID-19: from the Emergency Cardiovascular Care Committee and Get With The Guidelines-Resuscitation Adult and Pediatric Task Forces of the American Heart Association. Circulation 2020;141(25):e933-e943 